\title{
Contextos e padrões do uso indevido de benzodiazepínicos entre mulheres
}

\author{
Contexts and patterns of undue use benzodiazepine among women
}

Ana Rosa Lins de Souza ${ }^{1}$ Emérita Sátiro Opaleye ${ }^{1}$ Ana Regina Noto ${ }^{1}$
${ }^{1}$ Departamento de Psicobiologia,

Universidade de São Paulo. R. Botucatu 862, Vila Clementino. 04023-062 São Paulo SP.

anarosalls@yahoo.com.br
Abstract The undue use of benzodiazepines particularly among women has raised concern in the public health area. This qualitative study aimed at understanding the beliefs and values associated with undue use of benzodiazepines among women. Thirty-three participants (aged between 18 and 60) with a history of undue use of benzodiazepines in the past year were selected intentionally using specific criteria. The interviews were fully transcribed and subjected to content analysis using NVivo software. The majority of respondents reported use for longer than the recommended duration (median 7 years) and they purchased the drug with a medical prescription. Reasons for use most given were to deal with anxiety, to improve sleep and to "flee from problems." Even those who acknowledged the possibility of being addicted were not motivated to stop taking the drug. Medical supervision did not necessarily seem to influence the perception of risk of undue use of benzodiazepines and it appears as a factor favoring the maintenance of prolonged use.

Key words Anti-anxiety agents, Women, Substance-abuse, Qualitative research
Resumo O uso indevido de benzodiazepinicos, especialmente entre mulheres, tem despertado preocupação na área de saúde pública. Este estudo objetivou compreender qualitativamente crenças e valores associados ao consumo indevido dessa substância por mulheres. Foram entrevistadas trinta e três mulheres (18-60 anos) com histórico de uso indevido de benzodiazepínicos no último ano, selecionadas intencionalmente e por critérios. Os discursos foram transcritos e submetidos à análise de conteúdo com auxílio do software NVivo. A maioria das entrevistadas referiu tempo de uso bem superior ao recomendado (mediana: 7 anos) e compra com receita médica. Os motivos de uso mais citados foram diminuição da ansiedade, problemas de insônia e fuga dos problemas. Apesar de reconhecerem a possibilidade de dependência, esta não motivou a interrupção do uso. O acompanhamento médico não pareceu, necessariamente, estimular a percepção de risco dos benzodiazepínicos, sendo um fator que favoreceu a manutenção do uso prolongado.

Palavras-chave Ansioliticos, Mulheres, Uso indevido de substâncias, Pesquisa qualitativa 


\section{Introdução}

Os Benzodiazepínicos (BZD) estão entre os medicamentos psicotrópicos mais consumidos em diferentes países no mundo, principalmente para tratamento de transtorno de ansiedade e como indutores de sono. Em um estudo canadense, Cunningham et al. ${ }^{1}$ observaram que $8,4 \%$ já haviam feito uso de BZD, sendo o uso prolongado (por mais de 100 dias) referido por 3,5\%. Em Taiwan, Fang et al. ${ }^{2}$ observaram que $18,6 \%$ da amostra da "Pesquisa Nacional em Saúde" já haviam usado BZD. No Brasil, há carência de dados quanto ao uso dessas substâncias, embora o uso na vida sem receita médica tenha sido reportado por 5,6\% de entrevistados de 12 a 65 anos em estudo domiciliar realizado nas 108 maiores cidades brasileiras. Algumas subpopulações parecem mais vulneráveis ao uso abusivo, como por exemplo, mulheres, casadas, fumantes, de baixa renda, com algum transtorno ansioso e com idade média entre 50-71 anos ${ }^{1-6}$.

$\mathrm{O}$ uso indevido de BZD, definido como o uso sem supervisão médica ou em quantidades/prazos superiores ao preconizado para tratamento, tem sido crescente nos últimos anos e se tornado objeto de preocupação na área de saúde pública junto com outros medicamentos psicotrópicos ${ }^{78}$.

Para casos de insônia e/ou ansiedade, é recomendado que a utilização de BZD não ultrapasse quatro semanas ${ }^{9}$. No entanto, estudos têm mostrado vários casos de uso prolongado, por muitos meses ou até mesmo anos ${ }^{10-13}$. Alguns estudos recentes chegam a observar tempo de uso superior a 20 anos $^{4,5}$. Parr et al. referiram razões de uso prolongado associadas à eventos na vida, problemas de insônia e transtornos mentais, incluindo depressão e ansiedade ${ }^{5}$. Nesse sentido, o abuso e a dependência de BZD passam a ganhar relevância para a saúde pública. Os principais efeitos adversos envolvem diminuição da cognição, amnésia anterógrada, sedação, redução da coordenação, aumento do risco de acidentes, tolerância, assim como riscos de abuso e dependência ${ }^{14}$. O uso indevido de ansiolíticos também esteve associado a cerca de um terço das visitas às emergências hospitalares em decorrência de uso indevido de medicamentos nos Estados Unidos ${ }^{15}$, e ainda que com limitada evidência, estudos apontam para uma associação entre o uso de BZD e o aumento da mortalidade ${ }^{16}$.

Muitos pacientes que usam BZD por um longo tempo, adquirem a prescrição com médicos, mas carecem de informações acerca de seus ris$\cos ^{12}$. Além disso, apesar da legislação vigente exigir a retenção de uma receita médica pela drogaria, o controle de venda dos BZD também é falho. Noto et al., através de análises das prescrições de psicotrópicos, detectaram irregularidades e descuido no preenchimento das prescrições médicas, como indícios de falsificação ${ }^{17}$.

A maioria dos estudos recentes sobre BZD tem centrado atenção no uso entre idosos e suas consequências, tais como sedação excessiva, fraturas decorrentes de quedas e lentidão psicomotora ${ }^{18,19}$. Apesar de alguns desses estudos abordarem o uso entre mulheres idosas ${ }^{20}$, são menos frequentes os trabalhos sobre o comportamento de mulheres de outras faixas etárias. Da mesma forma, poucos são os estudos em outras faixas etárias que avaliam numa perspectiva qualitativa os discursos das usuárias de longo prazo de BZD ${ }^{21,22}$.

Diante da elevada prevalência de uso de BZD entre mulheres, bem como o potencial de abuso desses medicamentos, o presente estudo propõese a preencher uma lacuna importante sobre a compreensão de padrões e contextos de uso indevido desses medicamentos na população feminina, no sentido de fornecer subsídios para direcionamento de políticas de saúde no país. Sendo assim, o presente estudo teve por objetivo compreender os fatores que favorecem o uso indevido de benzodiazepínicos entre mulheres adultas (18-60 anos), com enfoque nas funções, contextos, percepção de risco e crenças atribuídas ao uso, no município de São Paulo e imediações.

\section{Métodos}

\section{Estudo}

A metodologia qualitativa foi escolhida por favorecer a compreensão em profundidade sobre padrões de uso indevido entre mulheres usuárias de BZD, sua percepção de risco e dependência. Este método permite estudar as crenças e os valores das pessoas em seus ambientes naturais, fornecendo informações sobre a maneira como compreendem e os contextos nos quais estão inseridas $^{23,24}$.

Para este estudo o uso indevido de BZD foi definido como o uso para insônia ou ansiedade por mais de quatro semanas, com prescrição em desacordo com a legislação vigente no Brasil (Portaria $344 / 98)^{25}$ ou sem acompanhamento médico. 


\section{Participantes}

Foi composta uma amostra intencional por critérios de mulheres entre 18 a 60 anos, com histórico de uso indevido de BZD no último ano, para insônia e/ou ansiedade, sem distúrbios cognitivos ou psiquiátricos evidentes e residentes no Estado de São Paulo.

Para iniciar contato com o universo pesquisado, foram realizadas entrevistas com informantes-chave, incluindo médicos psiquiatras, farmacêuticos e coordenadores de centros comunitários femininos. A partir destes, foram indicadas as primeiras entrevistadas. Em alguns poucos casos, a amostra seguiu pela técnica de snowball sampling ${ }^{26}$, mas a maioria das entrevistadas afirmou não conhecer ou não querer indicar outra pessoa. Dessa forma, a composição da amostra seguiu técnica mista, ou seja, a pesquisadora continuou intenso contato com as mais diferentes fontes, como rede de pessoas conhecidas, profissionais, centros comunitários, postos de saúde e sites de redes sociais. A composição da amostra seguiu até a saturação teórica para os aspectos mais relevantes do estudo, sendo encerrada com 33 entrevistas.

\section{Entrevistas}

A coleta de dados ocorreu por meio de entrevista semiestruturada. O roteiro de entrevista foi elaborado a partir de extensa revisão da literatura e realização de entrevistas piloto, com questões sobre: caracterização sociodemográfica da entrevistada, histórico e atual padrão do uso de BZD, funções do uso, percepção de risco à saúde, consequências do uso, formas de aquisição do medicamento, orientações médicas e farmacêuticas, estratégias de redução ou parada do consumo e crenças sobre o uso e dependência.

As entrevistas foram realizadas por uma única pesquisadora, a qual participou ativamente do projeto de pesquisa desde a concepção e a elaboração do roteiro de entrevista, bem como realizou cursos prévios de formação em pesquisa qualitativa. Ao longo do processo, a entrevistadora foi discutindo com outras pesquisadoras sobre o conteúdo das entrevistas para análise preliminar dos dados e orientação sobre a composição amostral.

As entrevistas duraram em média 1 hora e ocorreram em locais isolados, em consultórios, na residência das entrevistadas ou em centros de convivência. Todas as entrevistas foram gravadas na íntegra, com conhecimento e autorização da entrevistada. As entrevistadas foram informadas sobre a confidencialidade do estudo e assinaram o Termo de Consentimento. O estudo foi aprovado pelo Comitê de Ética em Pesquisa da Universidade Federal de São Paulo.

As entrevistadas foram identificadas por um código alfa-numérico, da seguinte forma: número da entrevista, inicial do primeiro nome, idade e tempo de uso do BZD, como por exemplo: Entrevistada 1, com o nome de Maria, com 23 anos e 5 anos de uso de BZD teria o código 1M.23.5.

\section{Análise dos dados}

Foram utilizados os referenciais da Análise de Conteúdo ${ }^{27}$ para análise dos dados. Todas as entrevistas foram transcritas na sua totalidade e, em seguida, foram submetidas à leitura cuidadosa com o intuito de criar as principais categorias de análise. As transcrições foram então inseridas no software QSR NVivo (versão 8) para a codificação dos dados e consequente categorização. Para garantir a validade dos dados foi realizado o processo de triangulação, no qual outra pesquisadora verificou a categorização realizada. As categorias de análise foram: motivos de uso, formas de aquisição, orientação médica, percepção de risco, percepção da dependência e razões para a não interrupção do uso.

\section{Resultados}

\section{Características das participantes}

Foram entrevistadas 33 mulheres entre $18 \mathrm{e}$ 60 anos de idade. A maioria $(n=20)$ se considerou pertencente à classe socioeconômica média. Em relação ao estado civil, 14 eram solteiras, 13 casadas, 5 separadas e 1 viúva.

A maioria $(\mathrm{n}=24)$ afirmou ser fumante ou ex-fumante. Uso na vida de bebidas alcoólicas foi referido por 23 mulheres, sendo que 12 já haviam feito uso concomitante com BZD. O uso na vida de maconha foi referido por 11 mulheres. Praticamente todas $(\mathrm{n}=32)$ as entrevistadas referiram uso na vida de outros medicamentos psicotrópicos, sendo a principal classe terapêutica, os antidepressivos $(\mathrm{n}=29)$. O uso na vida de BZD concomitante a outros medicamentos psicotrópicos foi referido pela maioria das mulheres $(n=28)$ e o uso de BZD associado aos anorexígenos foi relatado por sete.

O histórico de uso de BZD foi muito diversificado, variando de 50 dias a 37 anos (mediana $=$ 
7 anos). A grande maioria referiu acompanhamento médico $(\mathrm{n}=24)$ e aquisição do medicamento com receita apropriada $(\mathrm{n}=30)$. A maioria $(\mathrm{n}=14)$ referiu períodos alternados de parada de uso. Apesar de terem usado BZD no último ano, seis mulheres não estavam em época de uso quando foram entrevistadas.

\section{Motivos de uso}

Os motivos de uso estavam relacionados, predominantemente, à diminuição da ansiedade (por exemplo, sintomas de pânico, situações estressantes, como brigas familiares, problemas relacionados ao trabalho), problemas de insônia (dificuldade para dormir ou para manter o sono) ou "fuga dos problemas" (como exemplo, para enfrentar algum momento angustiante na vida, para dormir melhor, sendo o uso nesses casos esporádicos, geralmente uma vez por semana). A seguir são apresentados alguns relatos de diferentes motivações:

...então ela (médica) passou clonazepam pra eu poder dormir... eu não conseguia dormir, e até hoje se eu não tomar eu não durmo... (1T.49.11)

...quando você toma, você relaxa, fica gostoso... (5M.50.7)

Acho que me ajuda. Me ajuda muito...é uma fuga... Quando eu tô com problema, é pra esquecer do problema. (21I.18.5)

\section{Formas de aquisição do medicamento e orientação médica}

Foi observado que a principal especialidade médica prescritora, para as entrevistadas deste estudo, foi a de psiquiatras (21), seguida de ginecologistas (5), cardiologistas (4) e clínico geral (4). As entrevistadas muitas vezes eram consultadas por dois ou mais especialistas, nos quais conseguia a prescrição.

... Se eu vou no cardiologista, ele me dá, se eu vou neurologista ele me dá, se eu vou no meu ginecologista ele me dá, então os três sabem que eu uso (13M.58.15).

A grande maioria referiu ter um médico de referência $(\mathrm{n}=25)$, mas nem sempre precisava passar em consulta para obtenção da prescrição. Quatro mulheres relataram ter ficado sem qualquer acompanhamento em outros momentos e seis disseram estar sem acompanhamento atual, embora tenham tido em algum momento da vida. Duas afirmaram nunca ter tido acompanhamento. As sem acompanhamento relataram que adquiriam os medicamentos com parentes, médico amigo/conhecido, ou amigo/familiar que trabalha em locais onde possuem acesso fácil ao BZD ou a prescrições. Não houve relato de mulheres com aquisição de medicamento com receita em desacordo com a Portaria 344/9825.

Eu ganho (o medicamento), nunca tive receita (21I.18.5).

Com essa médica... fiquei dezesseis anos na Inter Clinica (plano de saúde), ela que me dava os medicamentos, aí depois que eu fui para Medial Saúde (plano de saúde) eu não pedi... Minha irmã passa no neuro há muitos anos, aí eu peço pra ela (16C.54.28).

As principais orientações dadas sobre os efeitos colaterais do BZD (não utilizar álcool conjuntamente com BZD, não dirigir sob efeito do ansiolítico e que o medicamento causa dependência) foram referidas por apenas cinco mulheres. Dessas, quatro foram orientadas por psiquiatras e uma por gastroenterologista. A orientação mais citada foi que poderia causar dependência $(\mathrm{n}=16)$.

O meu ginecologista, eu perguntei pra ele da dependência e ele falou: 'Olha do jeito que você está tomando, pelo teu perfil eu não acredito que vai te causar dependência' (25Y.46.4).

\section{Percepção de risco}

Menos da metade das entrevistadas $(\mathrm{n}=14)$ relatou percepção de risco frente ao seu consumo de BZD. As que fizeram alguma referência aos riscos citaram a possibilidade de causar dependência, "problemas na memória", diminuição/alteração de reflexos na direção de veículos e descoordenação motora. Nem todas tinham esse conhecimento por orientação médica, sendo que algumas relataram percepção de risco com base em experiência pessoal.

...porque o Rivotril ${ }^{\circledR}$ causa um problema de coordenação, né?... E eu tive muitas quedas em escadas... (23R.46.6)

A grande maioria das entrevistadas relatou despreocupação ou minimização dos riscos. Praticamente todas afirmaram preferir assumir os riscos para manter os benefícios do uso do BZD.

Eu acho que é uma dependência necessária... Eu penso em ter qualidade de vida, mesmo que isso encurte o tempo de vida, eu prefiro ter uma qualidade (2I.53.18)

Ah! Eu tomo há tantos anos e nunca fez mal, sei lá. Viciou, só viciou. Ele faz mal? (15A.48.3)

...mas se existe, se eu tenho acesso a ele e se ele não me faz mal, muito pelo contrário, não tem porque eu não tomá-lo [...] Eu acho que se o remé- 
dio tá aí pra me ajudar, ... fantástico, não tem porque eu me privar daquilo. (14J.26.10)

\section{Percepção da dependência}

A percepção da dependência relatada pelas usuárias foi observada através de experiência pessoal, por meio de sintomas em períodos de abstinência. Também foi referida a antecipação da falta do medicamento, por exemplo, pelo sentimento de angústia em situações nas quais os comprimidos estavam acabando e ainda não havia sido adquirida receita para nova compra. Muitas relataram não conseguir viver sem a medicação.

... Eu sinto a falta do medicamento quando eu já começo a ter aquelas sensações de nervosismo e irritabilidade... (31F.27.11)

Apesar do elevado tempo de uso, apenas 16 entrevistadas referiram a percepção da sua dependência, tendo como referência a dificuldade de dormir sem a medicação, sensação de "irritação" quando fica sem usar e de "desespero" diante da eventual falta da medicação.

Porque só ver o desespero que eu fico quando eu não tenho ele, já é um sinal de dependência... (7T.31.11)

Olha, sou dependente sim... só o fato de saber que não tem mais, já é uma coisa que vai me fazer não dormir... (7T.31.11)

Uma entrevistada, com histórico de dependência de maconha, comparou a dependência de BZD como pior:

O vício que eu tinha com a maconha era muito menor, muito menor, eu não tinha medo de ficar sem fumar, agora se acaba o remédio eu me desespero, eu tenho medo de ficar sem ele (7T.31.11)

Por outro lado, aquelas que não se consideravam dependentes, atribuíram os seguintes referenciais para justificar seu julgamento: não uso diário, conseguir ficar sem o medicamento ou dormir sem a medicação, usar com prescrição ou acompanhamento médico e a crença de ter controle sobre a quantidade consumida.

...Porque eu não uso todo dia é só quando eu to desesperada. (10G.25.4)

...Se eu fosse dependente eu acho que eu tomaria toda a noite em dez anos, eu não taria tomando só a metadinha... (9J.55.12)

...Eu tomo...quando eu acho que eu tenho que tomar... Ele é a muleta, eu vou parar, quando eu quiser... (4M.50.15)

\section{Razões para a não interrupção de uso}

A grande maioria das entrevistadas $(\mathrm{n}=28)$, mesmo aquelas que referiram já ter parado de usar o medicamento em algum momento da vida $(\mathrm{n}=12)$, não cogitavam a possibilidade de parar de usar o ansiolítico. Os principais motivos para a manutenção do uso estavam relacionados especialmente pelo receio de não conseguir dormir, pela reincidência dos sintomas ou por não perceber motivos para deixar de usar.

Porque se eu não tomar eu não consigo dormir... Eu tenho a consciência de saber que é muito tempo, mas enquanto eu não puder me libertar, eu vou tomando o remédio [...] só de eu pensar (em parar ou diminuir) as vezes eu já fico nervosa (16C.54.28)

... porque ele causa dependência, na própria caixa está falando e eu já tou sentindo que eu já tou dependente muito dele [...] por enquanto eu sinto que eu tenho que tomar (30T.47.4)

Porque eu não vejo necessidade de parar. Eu não acho que precise disso (14J.26.10)

\section{Discussão}

A principal forma de uso indevido observada neste estudo foi a de extrapolação do consumo por períodos extremamente longos. As principais funções de uso foram para redução de insônia, da ansiedade ou para lidar com problemas. O acompanhamento médico parece não garantir o conhecimento sobre os riscos do uso prolongado. Apesar da percepção da dependência, algumas mulheres ainda preferiam continuar usando, alegando ser necessário o uso e não perceber motivos para a sua interrupção.

A despeito das vantagens da pesquisa qualitativa no fornecimento de subsídios para a compreensão sobre o uso indevido de BZD, algumas limitações deste estudo devem ser observadas. As barreiras de identificação de mulheres que usam BZD podem ter excluído da amostra alguns perfis de usuárias de maior dificuldade de acesso, como mulheres de classes socioeconômicas muito elevadas. O autorrelato e o viés de memória também merecem ser considerados como possíveis limitações. Por se tratar de um estudo de abordagem qualitativa, no qual a amostra foi composta a partir de critérios específicos (amostra intencional), os resultados obtidos não podem ser generalizados.

O consumo de BZD no Brasil sofre influência de diversos fatores que vão desde a facilidade 
médica em receitá-los e a automedicação, até a popularização entre pares através do empréstimo e/ou indicação dos usuários para familiares ou amigos ${ }^{19}$. Além disso, características da nossa sociedade moderna como estresse no ambiente de trabalho, má remuneração, desmotivação e longas jornadas de trabalho também favorecem o uso de $\mathrm{BZD}^{28}$. No que tange ao gênero feminino, a necessidade de estar presente no mercado de trabalho não isenta a mulher de ainda ser a principal responsável pelas tarefas relativas ao lar e à família, ficando, portanto, mais suscetível a sintomas de ansiedade. Nesse aspecto, há uma internalização de valores estereotipados da figura feminina moderna como uma heroína, necessitando gozar de tranquilidade e bom sono para atuar bem nos seus papéis diários. Essa imagem foi amplamente explorada em propagandas da indústria farmacêutica quanto ao uso dos BZD e ainda hoje persiste no imaginário médico e popular. Cabe ainda ressaltar o peso que a indústria farmacêutica exerce na prescrição médica e consequentemente na prescrição de BZD através não apenas do incentivo publicitário de medicamentos, mas também por visitas de propagandistas, distribuição de brindes e de amostras grátis, dentre outras abordagens ${ }^{29}$.

Os motivos de uso, predominantes neste estudo, estiveram relacionados a transtornos de ansiedade, problemas de insônia, ou "fugas dos problemas". Esse achado, embora parcialmente enviesado pelos critérios de inclusão para uso indevido, estão de acordo com vários outros estudos. Mendonça et al. indicaram que as principais queixas descritas eram para insônia, ansiedade e nervosismo, bem como para lidar com conflitos familiares ${ }^{30}$. Esses autores discutem ainda que o papel da mulher de cuidadora contribui para o consumo de BZD diante das preocupações com os filhos, as discussões e as dificuldades econômicas. Elas assumem um papel no gerenciamento de conflitos familiares. Também consideram que dificuldades econômicas e conflitos podem contribuir para definir o modo e o prazo em que serão consumidos os BZD.

Paralelo a essas questões, Lader et al., em ampla revisão internacional sobre a descontinuação de BZD na rede básica de saúde, ressaltaram que os eventos adversos desses medicamentos são bem documentados e é crescente o número de pesquisas que questionam sua efetividade. Da mesma forma, o potencial para abuso e dependência dos BZD tem se tornado mais aparente e a descontinuação do uso deve ser incentivada pelo aumento na capacidade cognitiva e psicomotora dos usuários, especialmente entre idosos?.

Mesmo tendo a percepção de risco e da dependência, as mulheres não cogitavam a possibilidade de ficar sem usar o ansiolítico. Essa observação indica que a percepção de risco parece não ser um fator decisivo para a interrupção do consumo. Foi observado que os principais motivos referidos para não interromper o uso estavam relacionados especialmente ao receio de não conseguir dormir, receio da reincidência dos sintomas ansiogênicos ou por não perceber motivos para deixar de usar, corroborando com Laranjeira e Castro ${ }^{31}$. Zandstra et al. também encontraram que usuários crônicos de BZD têm baixa percepção da sua saúde geral e mental ${ }^{32}$.

Cook et al. discutiram que os pacientes usuários de BZD minimizam ou negam os riscos de potencial de abuso, uso inapropriado e efeitos físicos, ressaltando também que raramente os médicos informam sobre os efeitos negativos ${ }^{4}$. Os autores consideram que os pacientes minimizam o potencial de causar danos, alegando consumir em dose baixa, além de negar a dependência, demonstrando resistência na descontinuação do uso. Os pesquisadores descreveram ainda que, tanto médicos quanto pacientes, consideram os BZD mais efetivos que outras alternativas de tratamento; acreditam que a tentativa de interromper o uso poderia ser demorada e inútil, em função da resistência dos pacientes, limitado acesso à saúde mental e falta de acompanhamento psicológico.

Tesser e Barros discutiram o poder da "medicalização social” liderada pela biomedicina como uma forma legitimizada de cuidados que reduzem a abordagem terapêutica com reflexão sobre o modo de vida, diminuindo o potencial cultural das pessoas para lidar autonomamente com situações adversas. Em contrapartida, observa-se a crescente utilização de práticas alternativas que não são presentemente consideradas parte da biomedicina, mas que podem ser utilizadas em conjunto com esta, com outras abordagens ou individualmente. $\mathrm{O}$ autor relata que os benefícios da utilização de alternativas aos medicamentos expandem-se à recuperação da relação médico-paciente, com novas disposições e motivações, além de estimular que cada sujeito assuma a responsabilidade de sua própria saúde-doença, aumentando seu poder de tomada de decisões ${ }^{33}$.

Tácon et al. relataram que tratamentos alternativos como a meditação pode reduzir a ansiedade, trazendo benefícios para essas mulheres usuárias de $\mathrm{BZD}^{34}$. Além disso, há evidência cien- 
tífica substancial dos efeitos positivos da prática de exercícios físicos para tratamento de transtornos de ansiedade ${ }^{35}$. Especificamente sobre ioga, uma revisão sistemática mostrou pouco efeito estatístico pelas diferentes metodologias adotadas, mas conclui que os resultados são promissores e que outros estudos nesse sentido devem ser incentivados para uma avaliação adequada dos efeitos ansiolíticos desta modalidade específica $^{36}$.

Parr et al. descreveram que entre os principais fatores para a cessação do uso estão a consciência da mudança de estilo de vida, e que outras formas de estratégias não farmacológicas deveriam ser tentadas antes de iniciar tratamento com $\mathrm{BZD}^{5}$. Uma vez iniciado, entretanto, deve-se fornecer claro aconselhamento da importância de se evitar o uso prolongado.

Auchewski et al. ${ }^{37}$ relataram que somente $13 \%$ de adultos entrevistados durante aquisição de BZD referiu ter recebido todas as orientações sobre os efeitos colaterais, como diminuição da atenção, interação com álcool e risco potencial de dependência. Esses resultados indicam uma falha na assistência farmacêutica, e corroboram a falha na orientação médica observada neste estudo. Por outro lado, Anthierens et al. indicaram que o paciente não sabe informações sobre a medicação, mas também não faz questão de saber, alegando que a relação médico/paciente está baseada na confiança ${ }^{38}$.

A grande maioria das entrevistadas referiu ter acesso a algum médico e a aquisição do medicamento com receita apropriada. Orlandi e Noto descrevem que a prescrição médica é um fator importante na manutenção do uso crônico ${ }^{39}$, sendo frequente a aquisição por algum médico conhecido, que geralmente são amigos, vizinhos, familiares ou colegas de trabalho do usuário. Anthierens et al..$^{38}$ descreveram que os médicos sabem que a própria atitude de prescrever influencia o uso, porém acreditam que os BZD não causam sérios danos, além de justificar a prescrição como forma de compreensão do sofrimento do usuário. Também afirmam possuir pouco tempo para o atendimento e para a introdução de outras abordagens de tratamento, além de considerar a dependência aos BZD como desencadeada por dosagens altas e características de personalidade.

A percepção da dependência relatada pelas usuárias foi observada pela queixa do que sentem quando estão sem a medicação, ou pela antecipação da falta do medicamento, ou ainda pelo relato de não conseguir viver sem a medicação.
Esses dados corroboram o estudo de Anthierens et al., que relata que a dependência foi observada mesmo em doses terapêuticas, notando-se que o paciente carregava comprimidos consigo, e que usava uma dose superior ao habitual mediante antecipação de situações estressantes, além de relato dos pacientes que tinham "medo" que os sintomas (como insônia ou ansiedade) voltassem se eles interrompessem a medicação ${ }^{38}$.

Ao que parece, as pacientes não são devidamente informadas sobre os riscos do uso contínuo de BZD pelos médicos porque eles próprios têm uma dificuldade em mensurar esse risco na relação risco-benefício. Essa ambiguidade entre julgar como necessário e único recurso terapêutico o uso contínuo do medicamento contra um possível risco que venha a surgir, coloca o médico numa posição cômoda de não explorar alternativas terapêuticas. Por outro lado, dada a baixa qualidade nas condições de trabalho da maioria dos serviços de saúde, é possível que na prática não existam de fato alternativas, como por exemplo, acesso à terapia psicológica. Igualmente, mesmo as usuárias que apresentam suficiente conhecimento sobre os riscos de abuso e dependência tendem a minimizar esses riscos e esse comportamento reforça uma pressão sobre o médico para continuar recebendo a prescrição. Portanto, ao se pensar em modificar essa realidade, faz-se necessário uma ação conjunta desde os serviços de saúde ao fornecerem estrutura ao prescritor para que possa considerar outras práticas antes de prescrever um BZD, bem como educação continuada a esses profissionais para entenderem os riscos reais quanto ao uso contínuo de BZD e por fim, orientação aos pacientes para terem ciência dos riscos associados ao abuso e à dependência de um psicotrópico.

\section{Considerações finais}

Os resultados mostram que o uso indevido relacionado ao tempo prolongado vem acompanhado de ausência de informações adequadas sobre os riscos dos BZD, mesmo sob supervisão médica. Ressaltam a importância da orientação e acompanhamento adequado, como campanhas informativas que salientam a necessidade de ampliação da percepção de risco pessoal entre mulheres que fazem uso prolongado de BZD, bem como educação continuada para profissionais farmacêuticos e médicos. Ainda, faz-se necessário a elaboração de estratégias terapêuticas outras junto aos serviços de saúde que benefici- 
em as mulheres usuárias dessas substâncias com interesse em cessar o uso e também aquelas com sintomas de ansiedade que podem tentar outros recursos antes de iniciar o medicamento.

\section{Colaboradores}

ARL Souza, ES Opaleye e AR Noto participaram igualmente de todas as etapas de elaboração do artigo.

\section{Referências}

1. Cunningham CM, Hanley GE, Morgan S. Patterns in the use of benzodiazepines in British Columbia: examining the impact of increasing research and guideline cautions against long-term use. Health Policy 2010; 97(2-3):122-129.

2. Fang SY, Chen CY, Chang IS, Wu EC, Chang CM, Lin KM. Predictors of the incidence and discontinuation of long-term use of benzodiazepines: a population-based study. Drug Alcohol Depend 2009; 104(1-2):140-146.

3. Neutel CI. The epidemiology of long-term benzodiazepine use. Int Rev Psychiatry 2005; 17(3):189197.

4. Cook JM, Biyanova T, Masci C, Coyne JC. Older patient perspectives on long-term anxiolytic benzodiazepine use and discontinuation: a qualitative study. J Gen Intern Med 2007; 22(8):1094-1100.

5. Parr JM, Kavanagh DJ, Young RM, McCafferty K. Views of general practitioners and benzodiazepine users on benzodiazepines: a qualitative analysis. Soc Sci Med 2006; 62(5):1237-1249.

6. de las Cuevas C, Sanz E, de la Fuente J. Benzodiazepines: more "behavioural" addiction than dependence. Psychopharmacology (Berl) 2003; 167(3):297303

7. McCarthy M. Prescription drug abuse up sharply in the USA. Lancet 2007; 369(9572):1505-1506.

8. Miller P, Degenhardt L. The global diversion of pharmaceutical drugs series. Addiction 2009; 104(3): 333-334.

9. Lader M, Tylee A, Donoghue J. Withdrawing benzodiazepines in primary care. CNS Drugs 2009; 23(1):19-34.

10. Voshaar RC, Gorgels WJ, Mol AJ, van Balkom AJ, Mulder J, van de Lisdonk EH, Breteler MH, Zitman FG. Predictors of long-term benzodiazepine abstinence in participants of a randomized controlled benzodiazepine withdrawal program. Can J Psychiatry 2006; 51(7):445-452.

11. Ten Wolde GB, Dijkstra A, van Empelen P, van den Hout W, Neven AK, Zitman F. Long-term effectiveness of computer-generated tailored patient education on benzodiazepines: a randomized controlled trial. Addiction 2008; 103(4):662-670.

12. Kapczinski F, Amaral OB, Madruga M, Quevedo J, Busnello JV, de Lima MS. Use and misuse of benzodiazepines in Brazil: a review. Subst Use Misuse 2001; 36(8):1053-1069. 
13. Nardi AE, Freire RC, Valenca AM, Amrein R, de Cerqueira AC, Lopes FL, Nascimento I, Mezzasalma MA, Veras AB, Sardinha A, de Carvalho MR, da Costa RT, Levitan MN, de-Melo-Neto VL, SoaresFilho GL, Versiani M. Tapering clonazepam in patients with panic disorder after at least 3 years of treatment. J Clin Psychopharmacol 2010; 30(3):290293.

14. Authier N, Balayssac D, Sautereau M, Zangarelli A, Courty P, Somogyi AA, Vennat B, Llorca PM, Eschalier A. Benzodiazepine dependence: focus on withdrawal syndrome. Ann Pharm Fr 2009; 67(6): 408-413.

15. Substance Abuse and Mental Health Services Administration (SAMHSA), Center for Behavioral Health Statistics and Quality. Drug Abuse Warning Network, 2006: National Estimates of Drug-Related Emergency Department Visits. Rockville, MD: HHS Publication; 2008. (N. SMA 08-4339)

16. Charlson F, Degenhardt L, McLaren J, Hall W, Lynskey M. A systematic review of research examining benzodiazepine-related mortality. Pharmacoepidemiol Drug Saf 2009; 18(2):93-103.

17. Noto AR, Carlini EdA, Mastroianni PC, Alves VC, Galduróz JCF, Kuroiwa W, Csizmarc J, Costac A, Fariac MA, Hidalgoc SR, Assis D, Nappoa SA. Analysis of prescription and dispensation of psychotropic medications in two cities in the State of São Paulo, Brazil. Revista Brasileira de Psiquiatria 2002; 24(2):68-73.

18. Almeida OP, Ratto L, Garrido R, Tamai S. Fatores preditores e consequiências clínicas do uso de múltiplas medicações entre idosos atendidos em um serviço ambulatorial de saúde mental. Revista Brasileira de Psiquiatria 1999; 21(3):152-157.

19. Mendonca RT, Carvalho AC. The role of elderly female consumers of allopathic tranquilizers in the popularization of the use of these medicines. Rev Lat Am Enfermagem 2005; 13(Spec. N):1207-1212.

20. Huf G, Lopes C, Rozenfeld S. Long-term benzodiazepine use in women at a daycare center for older people. Cad Saude Publica 2000; 16(2):351-362.

21. Carvalho LF, Dimenstein M. A mulher, seu médico e o psicotrópico: redes de interfaces e a produção de subjetividade nos serviços de saúde. Interações 2003; 8(15):37-64.
22. Carvalho LF, Dimenstein M. O modelo de atenção à saúde e o uso de ansiolíticos entre mulheres. Estudos de Psicologia 2004; 9(1):1-3.

23. Pope C, Mays N. Métodos qualitativos na Pesquisa em saúde. In: Pope C, Mays N, editors. Pesquisa Qualitativa na atenção à saúde. Porto Alegre-RS: Artmed; 2009. p. 11-21.

24. Patton MQ. Qualitative evaluation and research methods. 2a Edição. London: Sage Publications; 1990.

25. Brasil. Anvisa. Portaria N.o 344, de 12 de maio de 1998. Aprova o Regulamento Técnico sobre substâncias e medicamentos sujeitos a controle especial. Diário Oficial da União 1998; 19 maio.

26. Biernarcki P, Waldorf D. Snowball sampling-problems and techniques of chain referral sampling. Sociological Methods and Research 1981; 10(2):141163.

27. Bardin L. Análise de Conteúdo. 4a Edição. Lisboa: Edições 70; 2009.

28. Firmino KF, Abreu MH, Perini E, Magalhaes SM. Factors associated with benzodiazepine prescription by local health services in Coronel Fabriciano, Minas Gerais State, Brazil. Cad Saude Publica 2011; 27(6):1223-1232.

29. Barros JA. (Mis)information on drugs: the double standard practiced by pharmaceutical companies. Cad Saude Publica 2000; 16(2):421-427.

30. Mendonça RT, Carvalho ACDd, Vieira EM, Adorno RdCF. Medicalização de mulheres idosas e interação com consumo de calmantes. Saúde e Sociedade 2008; 17(2):95-106.

31. Laranjeira R, Castro LA. Potencial de abuso de benzodiazepínicos. In: Bernik MA, organizador. Benzodiazepinicos, quatro décadas de experiência. São Paulo: Edusp: 1999. p. 187-198.

32. Zandstra SM, Furer JW, van de Lisdonk EH, Bor JH, Zitman FG, van Weel C. Differences in health status between long-term and short-term benzodiazepine users. Br J Gen Pract 2002; 52(483):805-808.

33. Tesser CD, Barros NF. Social medicalization and alternative and complementary medicine: the pluralization of health services in the Brazilian Unified Health System. Rev Saude Publica 2008; 42(5):914920.

34. Tacon AM, McComb J, Caldera Y, Randolph P. Mindfulness meditation, anxiety reduction, and heart disease: a pilot study. Fam Community Health 2003; 26(1):25-33. 
35. Wipfli BM, Rethorst CD, Landers DM. The anxiolytic effects of exercise: a meta-analysis of randomized trials and dose-response analysis. J Sport Exerc Psychol 2008; 30(4):392-410.

36. Kirkwood G, Rampes H, Tuffrey V, Richardson J, Pilkington K. Yoga for anxiety: a systematic review of the research evidence. Br J Sports Med 2005; 39(12):884-891.

37. Auchewski L, Andreatini R, Galduroz JC, de Lacerda RB. Evaluation of the medical orientation for the benzodiazepine side effects. Rev Bras Psiquiatr 2004; 26(1):24-31.

38. Anthierens S, Habraken H, Petrovic M, Deveugele M, De Maeseneer J, Christiaens T. First benzodiazepine prescriptions: qualitative study of patients perspectives. Can Fam Physician 2007;53(7):12001201.

39. Orlandi P, Noto AR. Misuse of benzodiazepines: a study among key informants in Sao Paulo city. Rev Lat Am Enfermagem 2005; 13(N. Spec.):896-902.

Artigo apresentado em 03/11/2011

Aprovado em 20/03/2012

Versão final apresentada em 27/03/2012 